\title{
uvby photometry of the chemically peculiar stars AR Aurigae, 3 Hydrae, 49 Cancri, and BX Bootis`
}

\author{
Saul J. Adelman \\ Department of Physics, The Citadel, 171 Moultrie Street, Charleston, SC 29409, U.S.A.
}

Received May 6; accepted July 10, 1997

\begin{abstract}
Differential Strömgren uvby photometric observations from the Four College Automated Photoelectric Telescope are presented for four Chemically Peculiar stars of the upper main sequence: the $\mathrm{HgMn}$ star binary AR Aurigae and the magnetic stars 3 Hydrae, 49 Cancri, and BX Bootis. AR Aurigae shows little variability except during the primary and secondary eclipses. The 11.305 day period of Maitzen et al. for 3 Hya is confirmed. A new period of 7.0501 days is found for 49 Cancri while a slightly refined period of 2.88756 days is derived for BX Boo.
\end{abstract}

Key words: stars: individual; AR Aurigae — stars: individual; 3 Hydrae — stars: individual; 49 Cancri stars: individual; BX Bootis — stars: chemically peculiar

\section{Introduction}

I present differential Strömgren photometry of the Mercury-manganese $(\mathrm{Hg}-\mathrm{Mn})$ binary star AR Aurigae and of the magnetic CP stars 49 Cancri, 3 Hydrae, and BX Bootis obtained during the six years (September 1990 - July 1996) of the 0.75-m Four College Automated Photoelectric Telescope (FCAPT) on Mt. Hopkins, AZ. These results are part of a continuing program (see, e.g. Adelman 1993) devoted to obtaining high quality periods and light curves in the four Strömgren bandpasses. Precise rotational period of magnetic CP stars are needed when detailed studies are made of these stars, for example of magnetic fields and abundance distributions in their photospheres. Comparison of light curves taken at different epochs are important for understanding these stars.

After the dark count, the telescope obtained measurements of the sky-ch $-c-v-c-v-c-v-c-c h-$ sky in each filter where sky is a reading of the sky, $c h$ that of

Send offprint requests to: S.J. Adelman

* Tables 2-5 will be available in electronic form at the CDS via anonymous ftp to cdsarc.u-strasbg.fr (130.79.128.5) or via http://cdsweb.u-strasbg.fr/Abstract.html
Table 1. Photometric groups

\begin{tabular}{llllll}
\hline Star & HD Number & Type & $V$ & $B-V$ & Spectral Type \\
\hline AR Aur & 34364 & $v$ & 6.14 & -0.06 & B9.5 V \\
18 Aur & 34499 & $c$ & 6.49 & +0.24 & A7 V \\
19 Aur & 34578 & $c h$ & 5.03 & +0.26 & A5 II \\
& & & & & \\
3 Hya & 72968 & $v$ & 5.72 & -0.03 & Ap1SrCrEu \\
HR 3437 & 73997 & $c$ & 6.63 & -0.02 & A1 Vn \\
HR 3342 & 71766 & $c h$ & 6.00 & +0.42 & F2 III \\
49 Cnc & 74721 & $v$ & 5.66 & -0.11 & A1pEuCr \\
34 Cnc & 72359 & $c$ & 6.46 & -0.02 & A1 IV \\
50 Cnc & 74873 & $c h$ & 5.87 & +0.11 & A1 V \\
BX Boo & 133029 & $v$ & 6.37 & -0.14 & B9pSiSrCr \\
47 Boo & 133962 & $c$ & 5.57 & -0.00 & A1 V \\
38 Boo & 130945 & $c h$ & 5.74 & +0.48 & F7 IV \\
\hline
\end{tabular}

the check star, $c$ that of the comparison star, and $v$ that of the variable star. Table 1 contains information on each group, a variable along with two supposedly non-variable stars, the comparison and check, against which the brightness of the variable is compared (Hoffleit 1982). Tables 2-5 present the data with their yearly means and standard deviations. Corrections were not made for neutral density filter differences among the stars of each group, each of which had one star with a different neutral density filter. The comparison and check stars were selected from supposedly non-variable stars in the vicinity of the variable on the sky that had similar $V$ magnitudes and $B-V$ colors. The standard deviations in Tables 2-5 of the check-comparison star differences indicate that these stars are constant at least for the period when they were observed.

For each magnetic CP star I plotted each variable's data using the best published period to see if the data approximately confirmed this period. Then I used the Scargle periodogram (Scargle 1982; Horne \& Baliunas 1986) and considered my data and those of other observers separately in calculating periodograms. If the periodograms 
AR Aur
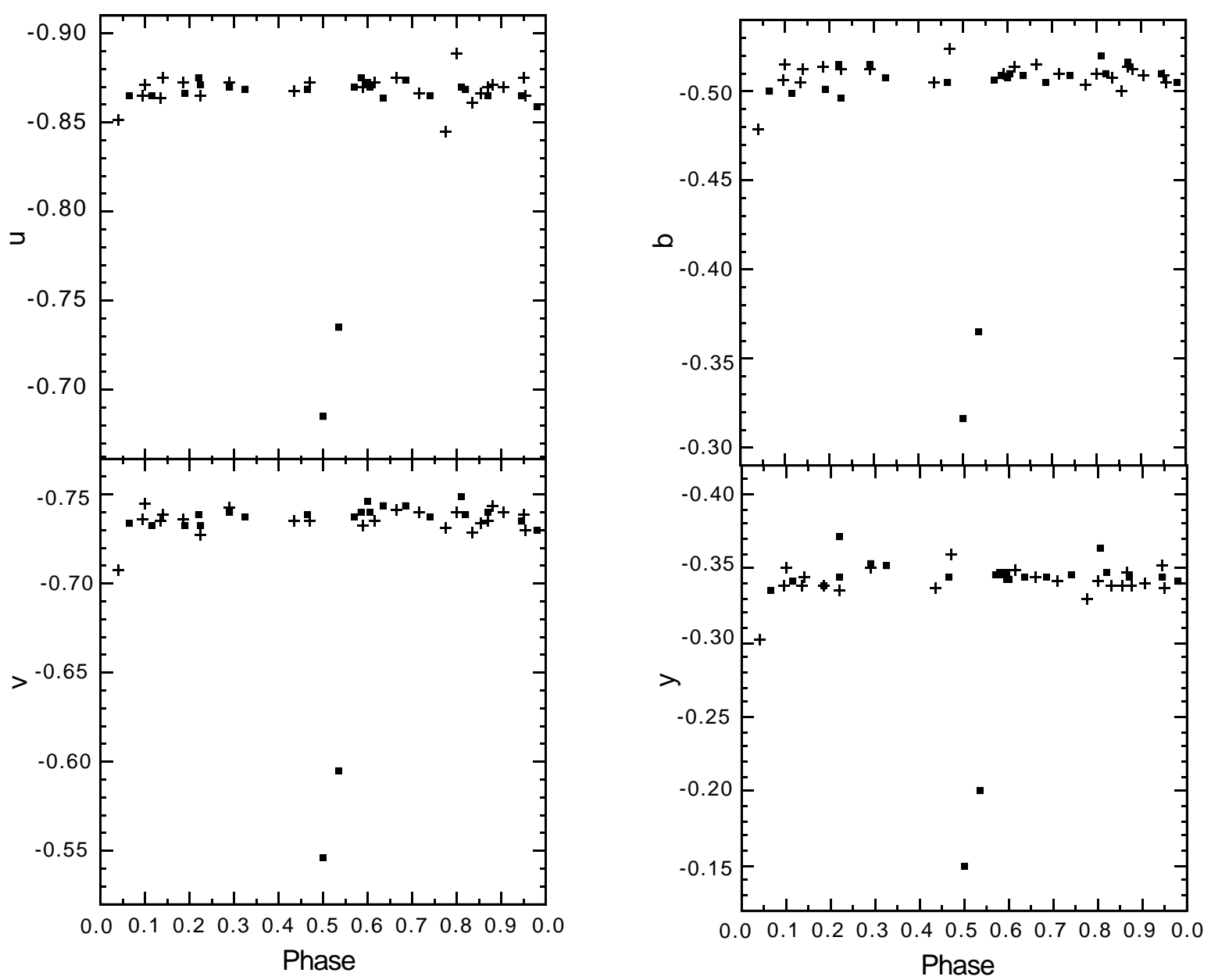

Fig. 1. FCAPT uvby photometry of the eclipsing HgMn binary star AR Aurigae plotted according to the ephemeris of Zverko et al. (1981). Solid rectangles are year 1 and pluses are year 2 values

confirmed the published period, then I adjusted the period to make all data coincide as well as possible in phase.

\section{AR Aurigiae}

AR Aurigae (=17 Aur = HD $34364=$ HR 1728) is a double lined eclipsing binary star (Popper 1959), both of whose components are $\mathrm{HgMn}$ stars. It is widely used as a standard to calibrate absolute parameters. Wyse (1936) determined the orbital elements of this spectroscopic binary system. Zverko et al. (1981) found evidence for a third object in this system. Johansen (1970) published photometry close to the Strömgen system.

In 1990-91 and in 1991-92, 25 and 23, respectively, sets of differential Strömgren values were obtained. Although the same check and variable stars were used, different neutral density filters were employed. Constant offsets were added to the year 2 values so that their values fit the trends of the year 1 values.
Figure 1, which is plotted using a single cycle, shows that there is very little variation in any color outside of the two eclipses which is in agreement with photometry of single HgMn stars (see, e.g., Adelman 1993). Excluding the eclipse observations, the $v-c$ standard deviations are similar to those found between two constant stars, of order 0.005 mag. The ephemerides used is that of Zverko et al. (1981) with a period of 4.134695 days and a correction for the effect of the third body. Near phase 0.5 we see the secondary eclipse. Year 1 values are indicated with closed rectangles and year 2 values with plus signs.

\section{3 Hya}

The magnetic CP star 3 Hydrae (= HR $3398=$ HD 72968 $=$ HV Hya) was studied most recently by Catalano \& Leone (1990) with observations from the Catania Observatory in their $U B V$ natural system. Previous photometry is by Wolff \& Wolff (1971), Maitzen et al. 


\section{Hya}
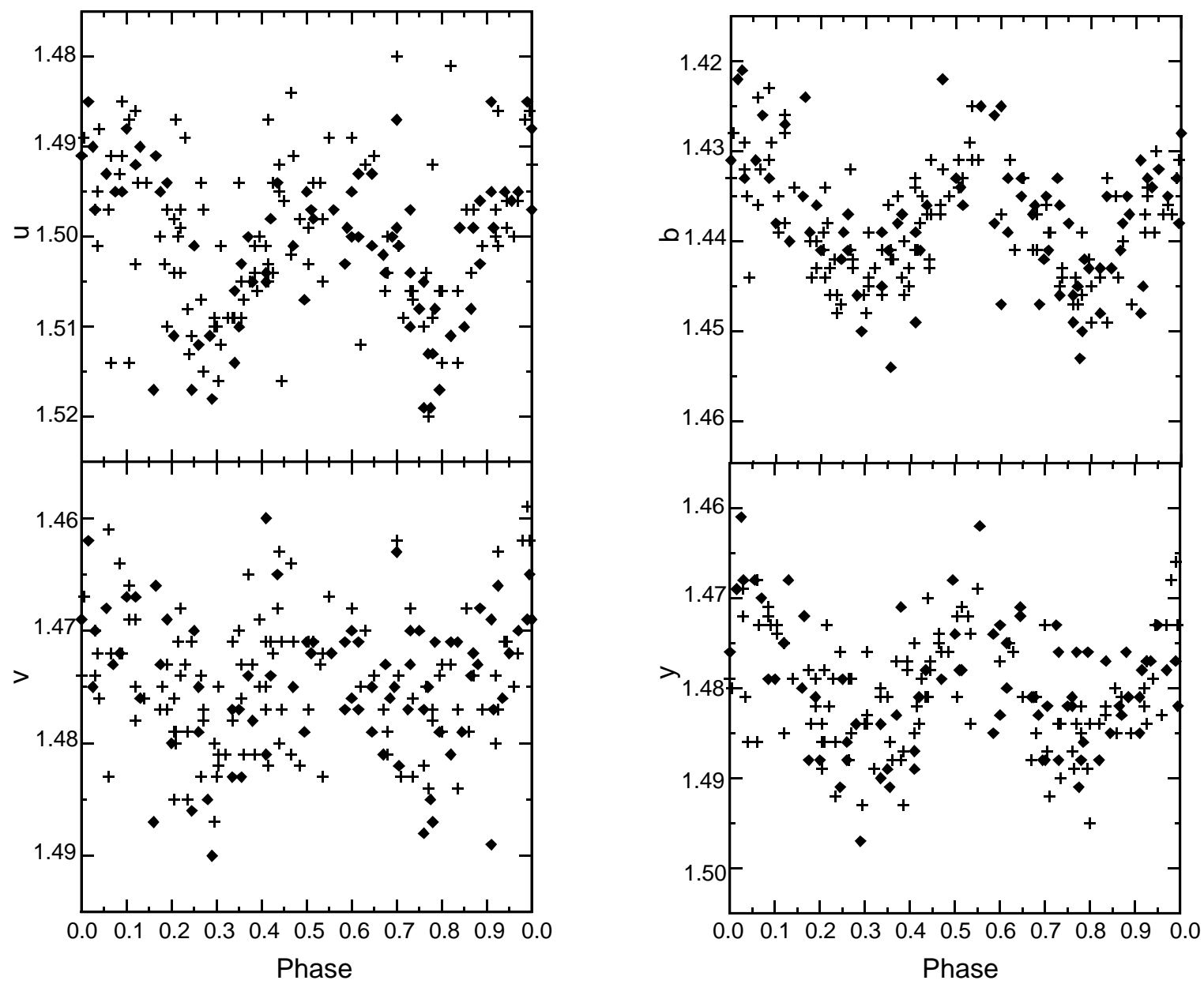

Fig. 2. FCAPT (pluses) and Maitzen et al. (1978) (closed diamonds) uvby photometry of the magnetic CP star 3 Hya using the elements of Maitzen et al. (1978)

(1978), and Heck et al. (1987). Wolff \& Wolff (1971) found a period of 5.57 days, but subsequent observers derived a double wave variation in all filters with approximately twice this period. Maitzen et al. (1978) found $\operatorname{HJD}\left(H_{\text {eff }}\right.$ maximum $)=24328997.68 \pm 0.23+$ $11.305 \pm 0.002 E$, elements which have also been used by subsequently published studies. When I plotted my FCAPT and previous uvby observations according to this ephemeris, then except for zero point differences in magnitude the shape and the amplitude of the variations in a given filter are very similar and the phase shift if any is small. This is a star with relatively small photometric variations.

Ten FCAPT observations were obtained in 1990-91, 1 in 1991-92, 11 in 1992-93, 26 in 1993-94, 28 in 1994-95, and 57 in 1995-96. Periodograms with my data alone gave a period close to that of Maitzen et al., 11.304 days. When I used a periodogram incorporating both the FCAPT and Maitzen et al. $u$ photometry the most likely period was
11.298 days. If correct it introduces a relative shift of 0.420 between my photometry and previous results according to the 11.305 day period. But as the light curve is symmetric either the Maitzen et al. period is correct or the relative phase shift is closer to 0.500 . As the long gap in time between my photometry and previous uvby values violates the assumptions of the periodogram method, the period is probably close to 11.305 days with the error in the period being \pm 0.001 day. Thus I will use the Maitzen et al. elements in comparing the observed light curves.

Figure 2 shows the FCAPT values as pluses and the previous uvby values from Maitzen et al. (1978) as closed diamonds. As there is a considerable amount of data, only one period is shown to simplify the figure. Corrections have been applied to the later data set to bring the mean values for each filter into agreement. The relative tightness of the photometry differs from filter to filter. The amplitudes are about $0.03 \mathrm{mag}$ in $u, 0.02 \mathrm{mag}$ in $v$ and 0.025 mag in $b$ and $y$. The clarity of the light curves is least for 


\section{Cnc}
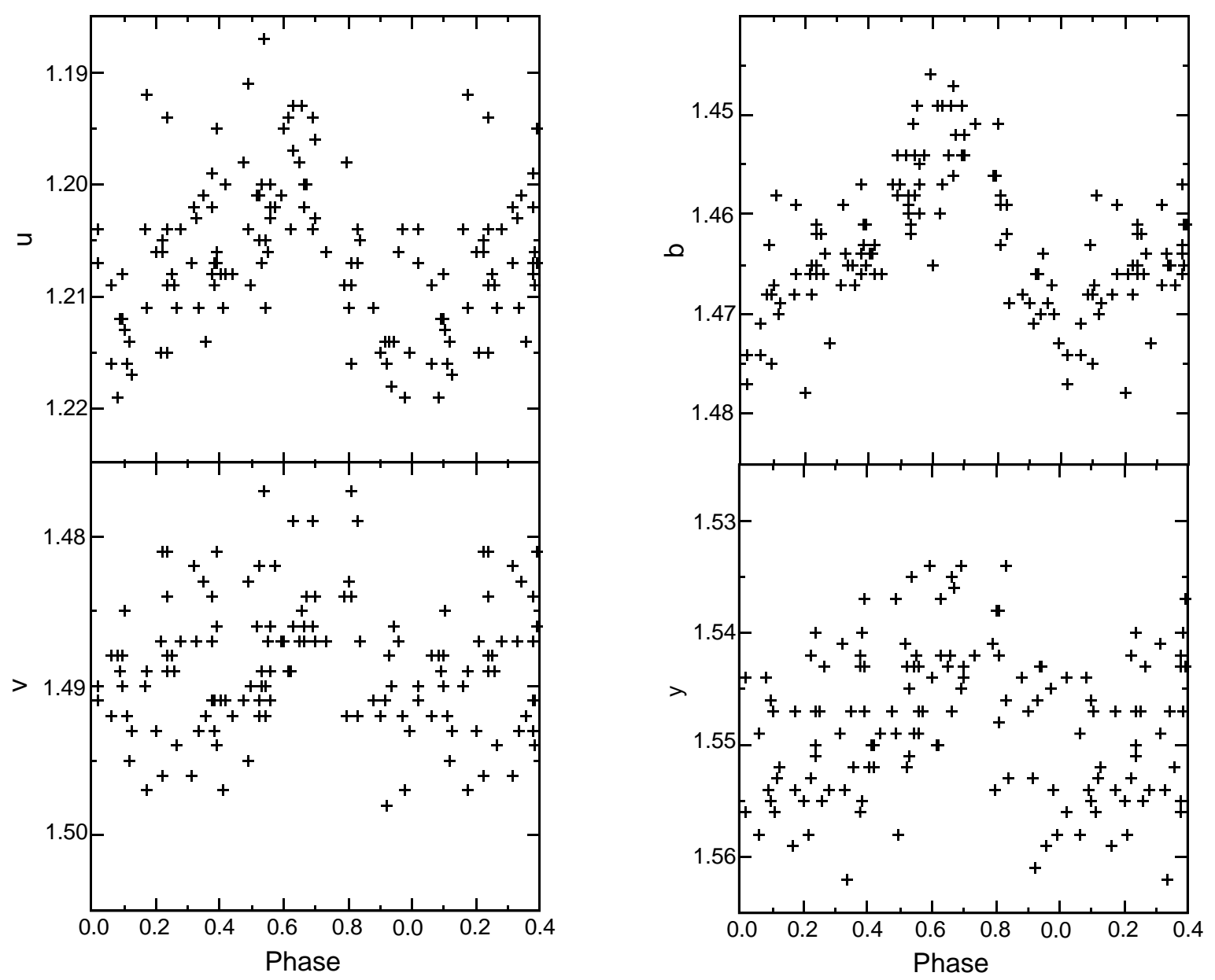

Fig. 3. FCAPT photometry of the magnetic CP star 49 Cnc plotted with this paper's ephemeris

$v$ and best for $b$. The small amplitudes and the quality of the photometry make it difficult to judge whether any changes in the shapes of the light curves are seen.

\section{49 Cancri}

Although Lanz \& Mathys (1991) found a 7.769 day period for the photometric variations of 49 Cancri $(=$ HR 3465 $=\mathrm{HD} 74521=\mathrm{BI}$ Cancri), they noted that this period needs to be confirmed. They used 54 measurements in the Geneva system distributed over almost 20 years with most values from 1987-88. Previous studies had indicated periods of $4.24 \mathrm{~d}$ (Rakosch \& Fielder 1974) and of $5.43 \mathrm{~d}$ (Stepien 1968). The largest amplitude found was about 0.03 mag.

There were 24 good sets of FCAPT uvby observations made in 1990-91, 11 in 1991-92, 4 in 1992-93, 26 in 199394 , and 35 in 1994-95. The amplitude of variability is $0.015 \mathrm{mag}$ in $v, 0.025 \mathrm{mag}$ in $u$ and $y$, and $0.030 \mathrm{mag}$ in $b$. A periodogram analysis of the $b$ magnitudes showed that the period was $7.0501 \pm 0.0002$ days. When the $V$ data of Lanz \& Mathys (1991) are plotted with this period, they show a single wave variation similar to the FCAPT data although a few points exhibit considerable scatter. I used the $u$ and $b$ light curves to define the zero phase as that of light curve minimum. As I began with the zero epoch of Catalano \& Leone (1993), I found $2447196.827 \pm 0.005$. The light curves (Fig. 3) are asymmetric with the rise to maximum taking longer than the fall to minimum. This was also noted by Catalano \& Leone who used the $7.769 \mathrm{~d}$ period. This suggests that there are at least two spots present, one of which is seen in the $b$ and perhaps in the $u$ photometry.

\section{BX Boo}

Wolff \& Morrison (1975) confirmed Winzer's (1974) discovery that the magnetic CP star BX Boo (= HD 133029 $=$ HR 5597) was variable with a period of 2.8881 days. The amplitudes of variation were 0.02 mag or less. Subsequent 


\section{BX Boo}
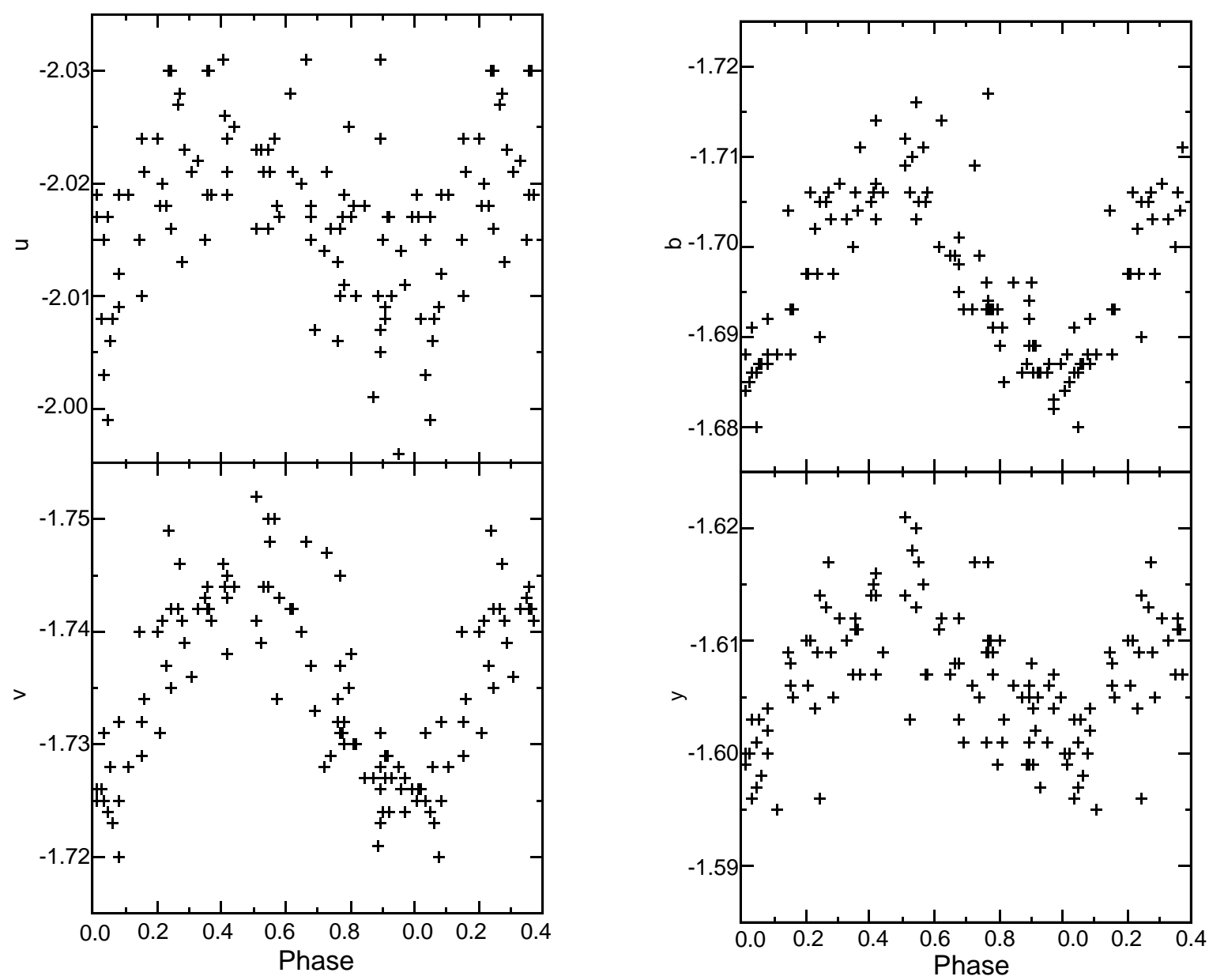

Fig. 4. FCAPT uvby observations of the magnetic CP star BX Boo plotted according to this paper's ephemeris

studies including those of Rakosh \& Fielder (1978) and Borra \& Landstreet (1980) have confirmed essentially this period.

Observations of BX Boo were made with the FCAPT (those in 1990-91 not being used in the analysis as they were made with choices of filter sets which are different from the set used subsequently). A periodogram analysis of this data showed that the period was $2.88756 \pm 0.00004$ days. As a phase zero point, I adopted 2448720.181 \pm 0.025 which is close to the light minimum and makes phase 0.50 the light maximum. It would be useful to have an earlier set of uvby photometry to improve the period, but Wolff \& Morrison did not publish their values. Although comparison with Winzer's (1974) photometry shows that there may be a phase shift, attempts to use this data to improve the period were not successful. In Fig. 4, I show the FCAPT photometry plotted according to my derived ephemeris for 1.4 periods. The single wave low amplitude light curves are similar to those presented by Winzer and Wolff \& Morrison. The amplitudes are 0.02 mag in $u$ and $y$ and perhaps 0.025 mag in $v$ and $b$.

\section{Final comments}

A new series of observations of the small amplitude variables 3 Hya, 49 Cnc, and BX Boo similar to those of this paper in a few years should be able to improve all the periods. Some of the scatter might be reduced by making many observations in a semi-continuous mode during the course of only a few nights now that the periods are sufficiently well known for that purpose.

Acknowledgements. This work was supported in part by NSF grant AST-9528506 to The Citadel and in part by grants from The Citadel Development Foundation. I appreciate the continuing efforts of Louis J. Boyd and Robert J. Dukes, Jr., to keep the FCAPT operating properly. 


\section{References}

Adelman S.J., 1993, A\&A 259, 411

Borra E.F., Landstreet J.D., 1980, ApJS 42, 421

Catalano F.A., Leone F., 1990, A\&AS 83, 491

Catalano F.A., Leone F., 1993, A\&AS 100, 319

Heck A., Mathys G., Manfroid J., 1987, A\&AS 37, 367

Hoffleit D., 1982, The Bright Star Catalogue, 4th edition, New Haven, Yale University Observatory

Horne J.H., Baliunas S.L., 1986, ApJ 302, 757

Johansen K.T., 1970, A\&A 4, 1

Lanz T., Mathys G., 1991, A\&AS 90, 365
Maitzen H.M., Albrecht R., Heck A., 1978, A\&A 62, 199

Popper D.M., 1959, ApJ 129, 659

Rakosch K.D., Fiedler W., 1978, A\&AS 31, 83

Scargle J.D., 1982, ApJ 263, 835

Stepien K., 1968, ApJ 154, 945

Winzer J.E., 1974, Ph. D. Thesis, University of Toronto

Wolff S.C., Morrison N.D., 1975, PASP 87, 231

Wolff S.C., Wolff R.J., 1971, AJ 202, 127

Wyse A.B., 1936, PASP 48, 24

Zverko J., Chochol D., Juza K., Ziznovsky J., 1981, IBVS 1997 